\title{
Need for Large-Area EDS Detectors for Imaging Nanoparticles in a SEM Operating in Transmission Mode
}

\author{
Steffi Rades ${ }^{1}$, Tobias Salge ${ }^{2}$, Roland Schmidt ${ }^{3}$ and Vasile-Dan Hodoroaba ${ }^{1{ }^{1 *}}$ \\ 1. BAM Federal Institute for Materials Research and Testing, Division 6.8 Surface Analysis and \\ Interfacial Chemistry, Berlin, Germany. \\ 2. Bruker Nano GmbH, Berlin, Germany. \\ 3. Hitachi High-Technologies Europe GmbH, Krefeld, Germany.
}

Accurate characterization of nanoparticles (NPs) with a high-resolution SEM with respect to morphology, shape and size distribution is carried out meanwhile routinely in more and more laboratories [1,2]. The valuable result of the metrological measurement of the NP size distribution -including traceability to the SI length unit - was also recently demonstrated by exploiting the transmission mode in a SEM, i.e. TSEM [1,3]. Especially for NPs prepared on thin foil supports like the typical TEM grids the transmission mode at low voltages (up to $30 \mathrm{kV}$ ) supplies a high imaging contrast so that sharp boarders of the NPs enable accurate lateral dimensional measurements of NPs size. There are just a few analytical methods which are able to characterize physico-chemical properties of individual NPs. In most cases a SEM has attached an EDS detector so that information on the elemental composition of the sample to be analyzed becomes possible. The addressed sample volume typically in the micrometer range for bulk specimens is considerably reduced if thin, electron transparent support films are used. Hence, one improves the spatial resolution however, at the cost of lower signal-to-noise ratios of the X-ray spectra, due to the tiny amount of substance excited [2,4,5]. One decisive technological development in the manufacturing of EDS detectors, namely the large-area SDD EDS, meanwhile available commercially in various designs, compensates this analytical drawback. If collection solid angles of 1-2 msr have been typically available for almost four decades, recent technological improvements in SDD EDS size and design have resulted in solid angles in the sr range.

The improved detection sensitivity of the large-area EDS detectors is of particular importance for NP analysis. Hence, EDX elemental imaging of single NPs down to about $10 \mathrm{~nm}$ and even smaller becomes possible, at least at a qualitative level. The door is open to the analysis of core-shell NPs structures. Moreover, both high sensitivity and high speed X-ray detection of the large-area EDS detectors make possible a good preservation of the NPs - many of them susceptible to e-beam damage - due to reduced time of analysis. Even if electrically non-conductive, most NPs deposited on conductive thin film supports, exhibit reduced charging effects. Working at low beam voltages in combination with large-area EDS detectors ensures both high spatial resolution and high detection sensitivity without being necessary to apply a conductive coating or working in low vacuum. The need and capabilities of low-energy detection will be highlighted by practical examples.

Figure 1 shows the limitations of the reduced collection angle offered by a typical $10 \mathrm{~mm}^{2}$ SDD EDS, however, yet indicating different elemental compositions of NPs of about 40-50 nm size, i.e. silica like and titania like, respectively. Figure 2 demonstrates the enhanced X-ray detection sensitivity by using a Flat Quad XFlash ${ }^{\circledR}$ 5060F EDS detector ( 1 sr collection solid angle) with respect to the presence of a carbon rich outer shell onto the fluorescent silica NPs. Note that not only the outer thin fluorescent dye shell is clearly detected, but also rests of the fluorescent dye on the carbon foil from sample preparation are visible. Such details where small density difference is available could be distinguished with high 
resolution SEM with transmission mode, however, no specific elemental information can be offered. In turn, a $10 \mathrm{~mm}$ SDD couldn't distinguish unambiguously such details.

\section{References:}

[1] E Buhr, N Senftleben et al., Meas. Sci. Technol. 20 (2009), p. 413.

[2] V-D Hodoroaba, S Benemann et al., Microsc. Microanal. 18 (Suppl 2) (2012), p. 1750.

[3] C Motzkus, T Macé, et al., J. Nanopart. Res. 15 (2013), p. 1919.

[4] V-D Hodoroaba, D Akcakayiran et al., Analyst (2014), in press.

[5] V-D Hodoroaba, S Rades et al., Surf. Interf. Anal. (2014), in press.

[6] The research leading to these results has received funding from the European Union's Seventh

Framework Programme (FP7/2007-2013) under grant agreement n 263147 (NanoValid - Development of reference methods for hazard identification, risk assessment and LCA of engineered nanomaterials).
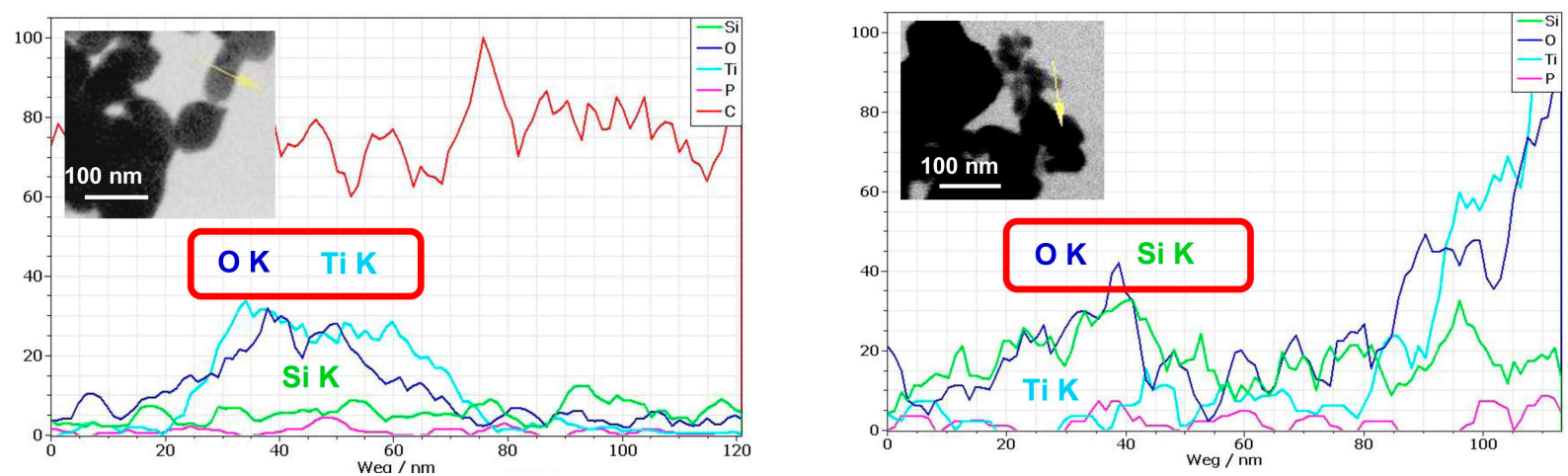

Figure 1. EDX linescans and TSEM images of silane coated titania. The positions of the linescans are marked in the T-SEM images with yellow arrows. Note the "titania like" particle shape in the left example and the "silica like" particle shape in the right example. The EDS used was a $10 \mathrm{~mm}^{2}$ SDD. Note the poor detection sensitivity due to the small solid angle, however clear differences in the chemical composition of the selected particles are recognizable.
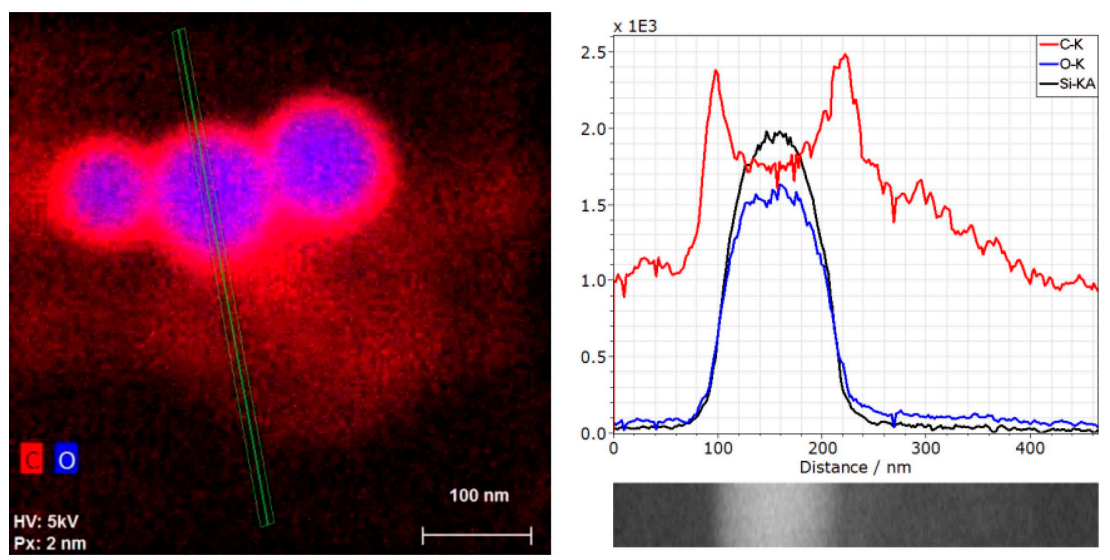

Figure 2. Composite EDX map of integrated peak intensities of carbon (red) and oxygen (blue) for fluorescent silica NPs taken with a Bruker XFlash QUAD EDS 5060F at a Hitachi SU8230 SEM at $5 \mathrm{kV}, 520 \mathrm{pA}, 22.5 \mathrm{kcps}$, 250x250 pixel, $2 \mathrm{~nm}$ pixel size and $377 \mathrm{~s}$; Right: EDX line scan (net intensities) extracted out of the spectrum image data cube corresponding to the distance marked in the EDX map (229 points, $467 \mathrm{~nm}$ length, $30 \mathrm{kcps}, 6.9$ s). Five adjacent pixel/spectra were binned for each point in order to improve impulse statistics. 